\title{
Mehr als das Begrünen der Wirtschaft
}

\author{
Bei der Betrachtung des „ganzen“ Wirtschaftens muss \\ der Care-Arbeit ein größerer Platz eingeräumt werden - \\ ihre Wertschöpfung entspricht immerhin der der Industrie, \\ des Handels, Gastgewerbes und Verkehrs zusammen. \\ Von Ulrike Röhr und Narges Lankarani
}

D ie Debatten über eine Green Economy - so unterschiedlich sie auch sein mögen - fokussieren gemeinhin auf die Wirtschaft und den Markt. Die Wirtschaft muss grüner oder ressourcenschonender werden, sie muss weiter wachsen - oder auch nicht, je nach Couleur des Autors oder der Autorin. Und der Markt ist das Regulativ für alles: von der gerechten Verteilung bis zur Bereitstellung der nötigen Infrastrukturen. Völlig ausgeblendet wird dabei, dass Wirtschaft mehr ist als die Güterproduktion, dass der gesamte Bereich der Sorgearbeit - neudeutsch: Care - die Grundlage jeglichen Wirtschaftens bereitstellt. Ohne Sorgearbeit keine Produktion, aber auch kein Wohlbefinden. So einfach diese Formel ist, so schwierig ist es, sie in ökonomische Konzepte zu integrieren. Die verengte Betrachtung auch der Green-Economy-Konzepte ist wenig geeignet, gesellschaftliche, soziale und kulturelle Aspekte des Wirtschaftsprozesses in den Blick zu nehmen. Konzepte für zukunftsfähiges (grünes oder nachhaltiges) Wirtschaften müssen sich aber daran messen lassen, ob sie die Trennungsstruktur zwischen produktiv und reproduktiv und die damit verbundenen Hierarchisierungen aufheben.

Die mehrheitlich von Frauen unbezahlt erbrachten Haushaltsleistungen tragen in erheblichem Umfang zur Wertschöpfung bei - werden aber nicht im Bruttoinlandsprodukt (BIP) berücksichtigt. Sie entsprechen in etwa der Bruttowertschöpfung der deutschen Industrie und der Bereiche Handel, Gastgewerbe und Verkehr zusammen (Statistisches Bundesamt, beruhend auf Zahlen von 2001). Allerdings geht die gesellschaftliche Bedeutung der Haus- und Familienarbeit weit über deren ökonomische Bedeutung hinaus.

Bei der Betrachtung des "ganzen“ Wirtschaftens rücken weitere Aspekte in den Fokus. Zum Beispiel, dass angesichts der zunehmenden Alterung der Gesellschaft der Pflegebereich einer der am stärksten wachsenden Bereiche ist. Darin zeigen sich dann die Grenzen der Effizienz: Güter können effizienter produziert werden, re-produktive Arbeiten - Pflege, Erziehung, Zuwendung - widersetzen sich häufig der Taktung in immer knappere Zeiteinheiten. Und schließlich verweist die Effizienzverliebtheit der meisten GreenEconomy-Konzepte auch auf deren gern vergessenen Gegenpart: die Suffizienz. Uta von Winterfeld beschreibt diese treffend als „Nicht immer mehr haben wollen müssen“.

Grundsätzlich stellt sich dabei nicht nur die Wachstumsfrage anders und erfordert eine differenzierte Diskussion darüber, was wachsen darf oder muss und was dagegen schrumpfen soll. Auch die Anerkennung und Bewertung der gesellschaftlich notwendigen Arbeiten muss neu geregelt werden, aber vor allem die (geschlechter-)gerechte Verteilung der bezahlten und unbezahlten Versorgungsund Pflegeleistungen.

Wohlbefinden ist ab einem bestimmten Einkommensniveau nicht mehr mit der Steigerung des Einkommens und des BIP verbunden, darauf verweist die rasant wachsende Anzahl an Publikationen zur
Glücksforschung. Dagegen spielt Gerechtigkeit eine bisher unterschätzte Rolle. Die für die Veränderung unserer Lebensstile, Konsum- und Produktionsmuster nötige gesellschaftliche und wirtschaftliche Transformation muss deshalb mit einer Umverteilung von Arbeit, Einkommen, Einfluss und Macht einhergehen; also mit Gerechtigkeit. Damit greift sie tief in bestehende und mit großem Beharrungsvermögen ausgestattete Strukturen ein. Deshalb muss dieser grundlegende Wandel zwangsläufig auf der Basis vielschichtiger, demokratischer Aushandlungsprozesse erfolgen. Diesen gesellschaftlichen Such- und Lernprozess zu gestalten, ist eine riesige Herausforderung. Die Alternative, ihn nicht über gesellschaftliche Partizipation zu gestalten und die Transformation von oben zu diktieren, hieße aber, sie zum Scheitern zu verurteilen.

Bei der Rio+20-Konferenz Ende Juni 2012 wurde das Konzept einer Green Economy von vielen Ländern des globalen Südens unter anderem mit dem Verweis auf eine neue Form der Kolonialisierung abgelehnt. Mehr noch, auch das Konzept der Care-Ökonomie wird von Frauenorganisationen aus dem Süden als eines westlicher Genderexpert(inn)en kritisiert, das der in den Ländern des globalen Südens schwachen Trennung zwischen Erwerbstätigkeit, Subsistenzwirtschaft und Versorgungsarbeit nicht gerecht wird. Das zeigt, dass es auch international dringend der Verständigung über tragfähige Konzepte bedarf.

\section{Literatur}

Schäfer, W. (2004): Unbezahlte Arbeit und Bruttoinlandsprodukt 1992 und 2001. In: Statistisches Bundesamt (Hrsg.): Auszug aus Wirtschaft und Statistik 9/2004. Wiesbaden.

I AUTORINNEN + KONTAKT

Ulrike Röhr ist Bauingenieurin und Sozialwissenschaftlerin und neben der Green Economy schwerpunktmäßig mit den Genderaspekten in Klima- und Energiepolitik befasst.

Narges Lankarani, Geografin, bereitet bei LIFE e.V. einen internationalen ExpertInnenworkshop zu Gender Green Economy und Care Economy vor.

E-Mail: roehr@life-online.de, lankarani@life-online.de, www.genanet.de/greeneconomy.html 
Copyright (C) 2013, IÖW und oekom Verlag. Die Nutzung des Artikels ist Abonnenten von Ökologisches Wirtschaften vorbehalten. Nachdruck und Vervielfältigung des Artikels einschließlich Speicherung und Nutzung auf optischen und elektronischen Datenträgern nur mit Zustimmung der Redaktion von Ökologisches Wirtschaften (http://www.oekologischeswirtschaften.de). 